\title{
A journal of real peak recognition of electrocardiogram (ecg) signals using neural network
}

\author{
Tarmizi Amani Izzah ${ }^{1}$, Syed Sahal Nazli Alhady ${ }^{1}$, Umi Kalthum Ngah, Wan Pauzi Ibrahim ${ }^{2}$ \\ ${ }^{1}$ School of Electrical Electronics Engineering, Universiti Sains Malaysia (Engineering Campus), Nibong Tebal, SPS, Penang \\ ${ }^{2}$ School of Medical Sciences, Universiti Sains Malaysia (Health Campus), 16150, Kubang Kerian, Kelantan
}

\section{Email address:}

izzah.amani@ymail.com (I. A. Tarmizi), sahal@eng.usm.my (S. S. N. Alhady), eeumi@eng.usm.my (U. K. Ngah), wpauzi@kb.usm.my (W. P. Ibrahim)

\section{To cite this article:}

Tarmizi Amani Izzah, Syed Sahal Nazli Alhady, Umi Kalthum Ngah, Wan Pauzi Ibrahim. A Journal of Real Peak Recognition of Electrocardiogram (ECG) Signals Using Neural Network. American Journal of Networks and Communications, Vol. 2, No. 1, 2013, pp. 9-16. doi: 10.11648/j.ajnc.20130201.12

\begin{abstract}
This paper describes about the analysis of electrocardiogram (ECG) signals using neural network approach. Heart structure is a unique system that can generate ECG signals independently via heart contraction. Basically, an ECG signal consists of PQRST wave. All these waves are represented respective heart functions. Normal healthy heart can be simply recognized by normal ECG signal while heart disorder or arrhythmias signals contain differences in terms of features and morphological attributes in their corresponding ECG waveform. Some major important features will be extracted from ECG signals such as amplitude, duration, pre-gradient, post-gradient and so on. These features will then be fed as an input to neural network system. The target output represented real peaks of the signals is also being defined using a binary number. Result obtained showing that neural network pattern recognition is able to classify and recognize the real peaks accordingly with overall accuracy of $81.6 \%$ although there might be limitations and misclassification happened. Future recommendations have been highlighted to improve network's performance in order to get better and more accurate result.
\end{abstract}

Keywords: Heart, ECG Signal, Features Extraction, Neural Network And Matlab Simulation

\section{State-of-the Art}

Neural network nowadays has been applied extensively in wide areas including classification, detection, aerospace, forecasting, heart diagnosis and many more. This project applied neural network method in analysing cardiac rhythms. Recognition of real peaks of ECG signals is important to diagnose the heart diseases. Doctors obtained ECG data from Holter device that recorded patient's heart beat and they perform analysis manually based on the waveform characteristics. Currently, cardiologist or doctors identify the real peak based on their knowledge and previous experiences. Some ECG signals easily obtained the real peak by looking at the waveform pattern but there is also some signals which is very difficult to identify their real peak. This manual identification might contain inaccuracy and small percentage of error. In addition, it could be a tedious way especially when analyzing a very low frequency of ECG signals. Doctors normally need an adequate time to study and verify the ECG waveform before getting the correct result for every patient examined. This works done defi- nitely time-consuming and not the efficient way.

Thus, this idea comes up that could be beneficial to cardiologists to recognize the real peak using new approach which is neural network. Neural network has the ability to memorize the pattern and directly gives the result accordingly. This eases the doctors and analysis could be done in way that is more efficient. Neural network also exhibits independent behavior as well as self-learning. It designed in such a way that exposed to enough training until it comes to a generalization state. Generalization means the network can memorize the data pattern and able to give result correctly based on the previous learning given. The system then tested to see its accuracy and performance.

It is then becoming a good momentum to exhort improvement in electrocardiography by offering a reliable as well as comprehensive solution for better ECG diagnosis [14]. Besides, by using neural network method, things will be much simpler, time-savings as well as reducing the needs of human efforts as machine has been trained to perform the desired workload. 


\section{Introduction}

The needs of technology and computerized analysis usage has exhorted researchers, professionals, engineers and other expert people combining their efforts together in implementing quality diagnosis tools. The term quality has been interpreted as easier and faster analysis, lack maintenance, high efficient as well as low in the cost. Due to that, another approach to analyse ECG signals has been chosen by using neural network method via matlab software, as matlab is well known with multifunction and powerful computerized tool software. This project has applied neural network approach to analyze ECG signals focusing on real peaks recognition since it provides valuable information to doctors regarding heart diagnosis. Recognition of real peak correctly is absolutely essential as it indicated the condition of heart as well as reflects to its functionality.

\subsection{Generation of ECG Signal}

The corresponding part in the heart plays their respective roles. Sinoatrial (SA) node will excite the beats that caused heart muscles to contract. Below is shown the location of Atrioventricular (AV) node and SA node which are responsible for generating ECG signal in the human's heart.

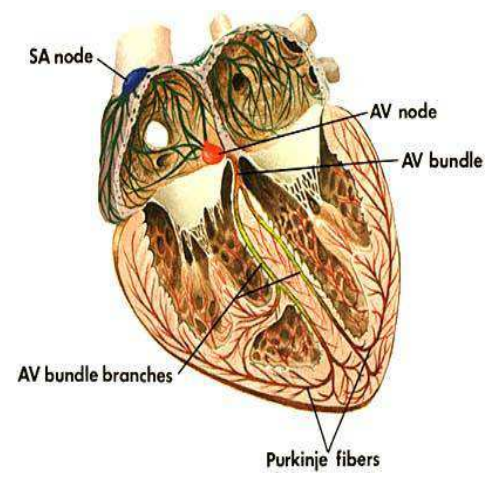

Figure 1. Location of SA and AV node [1].

The contraction of heart's muscles soon will be recorded as an electrical activity of the heart called ECG signal. Based on the pattern of ECG recording, heart status could be identified whether possessed of any cardiac arrhythmias or otherwise. As known, heart muscle possessed the characteristic of depolarization and repolarization. Depolarization is referred to the electrical potential activity excited by heart muscles while repolarization is a relaxation state when the heart changing back to its original position. $\mathrm{P}$ wave generated due to the atrial depolarization, QRS complex represented ventricular depolarization while $\mathrm{T}$ wave represented ventricular repolarization [2]. Figure below showed the corresponding part of heart function with respect to the ECG signal obtained.

Abnormalities happened in the respective waves segment will provide ideas to doctors and cardiologists at where the part of heart is having problems.

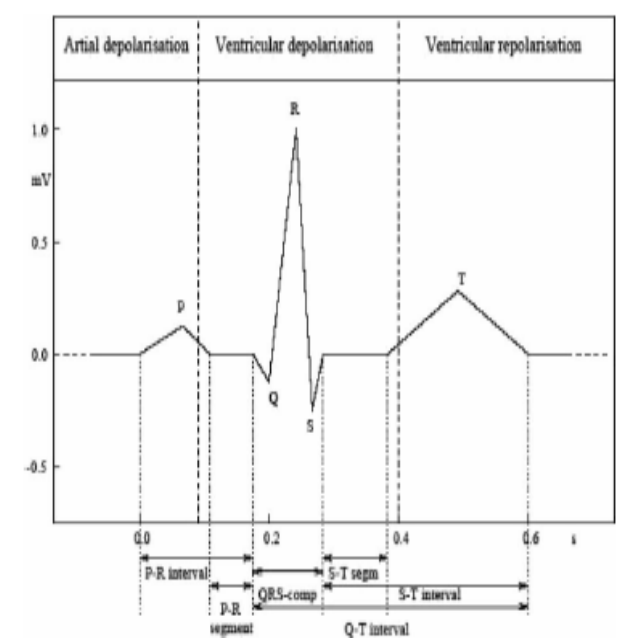

Figure 2. ECG signal based on heart function [3], [4].

\subsection{Neural Network}

A neural network is a type of computational model which is able to solve multi problems in various fields. It processes the information in a similar way as the human brain concept processing the information [5]. Basically, neural network consists of large processing elements called neurons working together to perform specific tasks. As in the human brain, there are thousands of dendrites which contain information signals. They transmitted the signals to the axon in the form of electrical spikes. The axon then sends the signals to another dendrites causing to a synapse. This synapse occurred when excitatory input is sufficiently large than the inhibitory input, and this concept of signal transmission also depicted on how neural network process inputs received. Figure below shown dendrites related structures for clearer understanding.

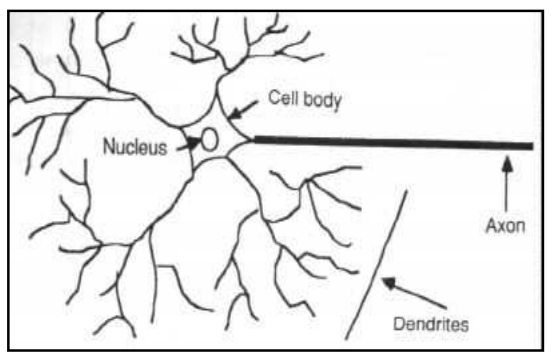

Figure 3. Dendrites [6].

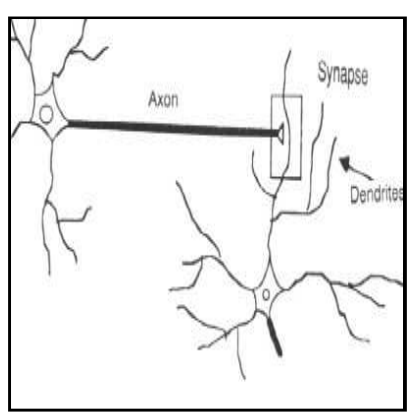

Figure 4. Synapses [6]. 
In neural network, dendrites carrying signals can be analogy as multiple inputs collected together for summation. Then, the combined inputs will be activated by activation function. Inputs that exceed threshold value will be feed to the output layer for final processing. During processing stage, inputs will be trained to produce desired target outputs until it come to a generalization stage. Generalization means at a condition where the network is able to recognize the inputs and the corresponding targets after undergone the training given. Then, the network will be tested by given new inputs signal to evaluate its performance and to see how accurate the output produced will be comparing to the target. Figure below depicted the analogy of human brain concept process the information to the neural network system.

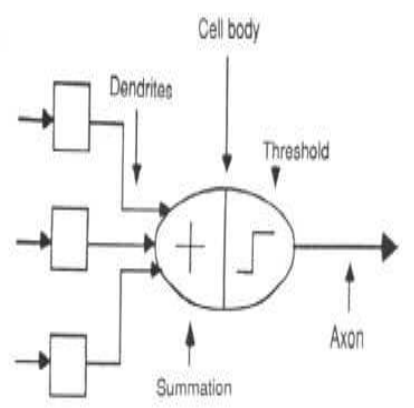

Figure 5. Neuron model [6].

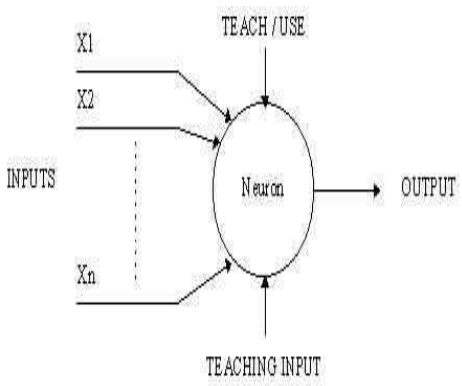

Figure 6. Neural networks [6].

Neural network consists of several architectures, from simple structure until the complicated ones.

\subsubsection{Single-Layer Feed forward Networks}

This is the simplest form of network architecture with only single layer output without any hidden layer. An input layer of source nodes will directly projects onto the output layer of neurons or computation nodes, just in one way butnot vice versa. Single layer is referring to the output layer which is just single output and not considered the input layer of source nodes since no computation performed there [7]. Figure 7 showed the corresponding figure including label.

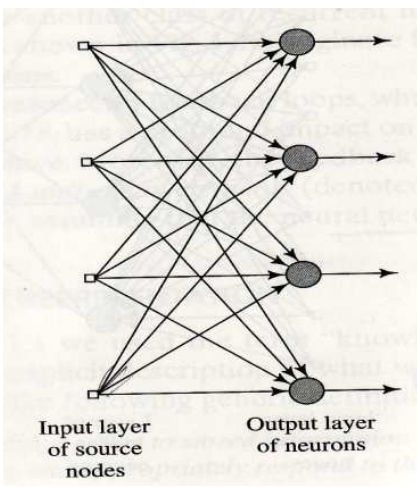

Figure 7. Single layer feedforward networks [7].

\subsubsection{Multilayer Feed-forward Networks}

This second layer is differ from above since it has one or more hidden layers. The computation also takes place in these hidden nodes. Hidden nodes are also used to intervene between the external input and the network output with respect to the network's manner. The structure of multilayer feedforward networks with one hidden layer as in figure 8 .

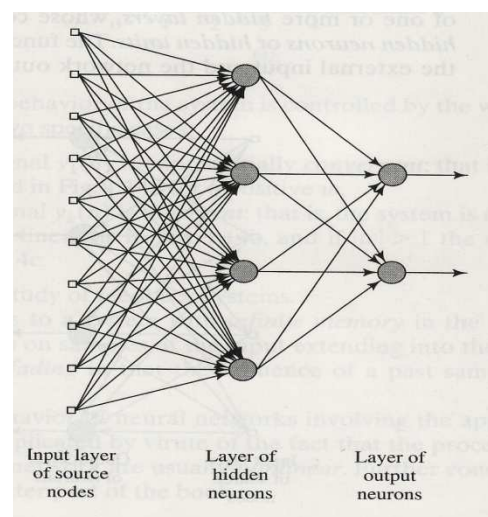

Figure 8. Typical of multilayer feedforward networks [7].

\subsubsection{Recurrent Network with No-Self Feedback Loops and No Hidden Neurons}

A recurrent neural network has at least one feedback loop. It may consist of a single layer of neurons with each neuron feeding its output back to all the input neurons as illustrated in figure 9. The feedback loops increased the learning capability of the network and on its performance. Besides, these feedback loops are also associated with unit delay elements $\left(\mathrm{z}^{-1}\right)$ which result in a nonlinear dynamical behavior in a condition when neural network contains nonlinear units. 


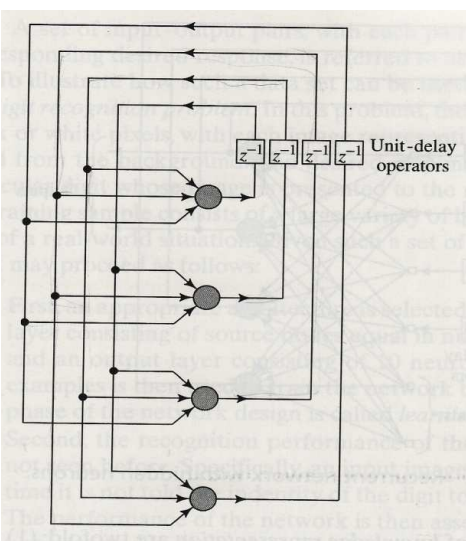

Figure 9. Typical of multilayer feedforward networks [7].

\subsubsection{Recurrent Network With Hidden Neurons}

This structure distinguishes itself from part (iii) with hidden neurons. The feedback connections originate from the hidden neurons and also from the output neurons. The structure illustrated in figure 10.

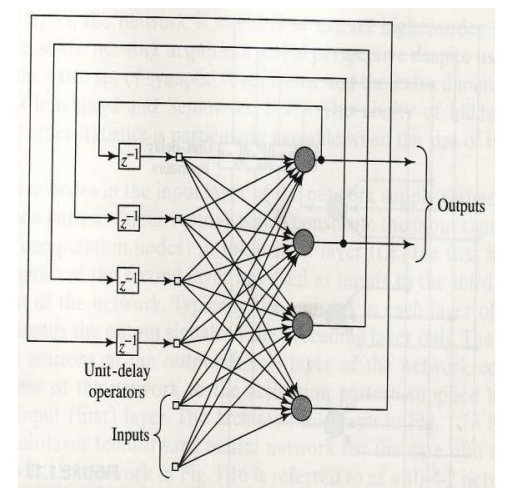

Figure 10. Typical of multilayer feedforward networks [7].

In neural networks, the input layer is passive while the hidden nodes and output layer are active and normally been activated by transfer function. These active nodes will modify weight and bias values to an optimum number where the network is best works at.There are many activation functions can be applied such as radial basis (radbas), competitive transfer function (compet), positive linear (poslin), saturating linear transfer function (satlin) and many more. The most commonly used is including hard-limit transfer function, linear transfer function and log-sigmoid transfer function. Neural networks need to be trained with suitable learning algorithm training functions corresponding to a network type. Among of the training functions are gradient descent backpropagation (traingd), Levenberg-Marquadt backpropagation (trainlm), gradient descent with adaptive learning rule backpropagation (traingda), random order incremental training with learning functions (trainr) and so on.

This experimental works used feed forward neural network with sigmoid hidden nodes and output neurons. It has been trained by scaled conjugate gradient backpropagation (trainscg) learning algorithm. Above are selected since they are most commonly used in various application, suitable for this project purpose and expected to be much efficient.

\subsection{Methodology}

Features extraction of ECG signals have been done to collect necessary data required for ECG analysis using neural network. In a truth fact, hundreds of input features could be extracted from the ECG signal. If all of the features are taken into consideration, identification information provided would be irrelevant and some do not give much significant to the network. Furthermore, the training duration also will be much longer. Neural networks also adaptive to a non-linear and irrelevant data with a degree of tolerance. Yet, their performance will be highly efficient when giving only appropriate and selected inputs [8].

First of all, several types of ECG signals obtained from healthy and unhealthy patients. Basically, ECG signal contains of PQRST peaks. In order to detect the peak correspondingly, an ECG signal has been divided into three segments. The first segment is $\mathrm{P}$ waveform, the second segment is QRS waveform and the last one is $\mathrm{T}$ waveform. $\mathrm{U}$ waveform is somehow exist in ECG signal, but it can be ignored as it does not really make any significant in cardiac diagnosis. The respective waveform after separating from the original signal is shown below.

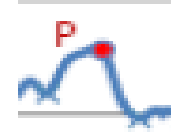

Figure 11. $P$ waveform.

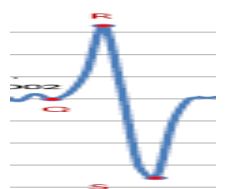

Figure 12. QRS waveform.

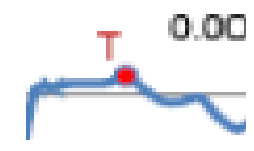

Figure 13.T waveform.

The next stage is extracting important data features and characteristics of those waveforms using manual computation. The manual computation has been verified and approved by cardiologist, but it is more recommended to use LabVIEW software to perform automated extraction in order to get accurate values and precise computation. These features selected are amplitudes, durations, gradients and polarity. Then, all the values will be normalized within the range from 0 to 1 only. All of these features then fed into the neural network system as its inputs with certain desired target defined. The ECG data signals are collected and arranged in the form of numbers. There are 20 types of ECG signals involved in this experimental simulation with 49 
samples (17 samples of $\mathrm{P}$ wave, 20 samples of QRS wave and 12 samples of $\mathrm{T}$ wave).Then, the simulation to get ECG waveform was performed by using Microsoft Excel for easier further analysis. Below is shown typical example of ECG signals and how features extraction is being accomplished. The same method of features extraction then been applied to the rest of ECG signals. This computation is quite time consuming and thus better system for extraction analysis is highly recommended to be implemented in the future. Referring to this figure below, real peak has been marked red in colour. The process to identify real peak is via template matching and cardiologist verification. The examined signal is compared with the source or template image. Those waveforms possessed high similarity in their morphological attributes and features will be classified accordingly.

Some of the waveforms whichever not have source template will get verified by cardiologist after marking the expected real peak based on theoretical knowledge or peak criteria's.

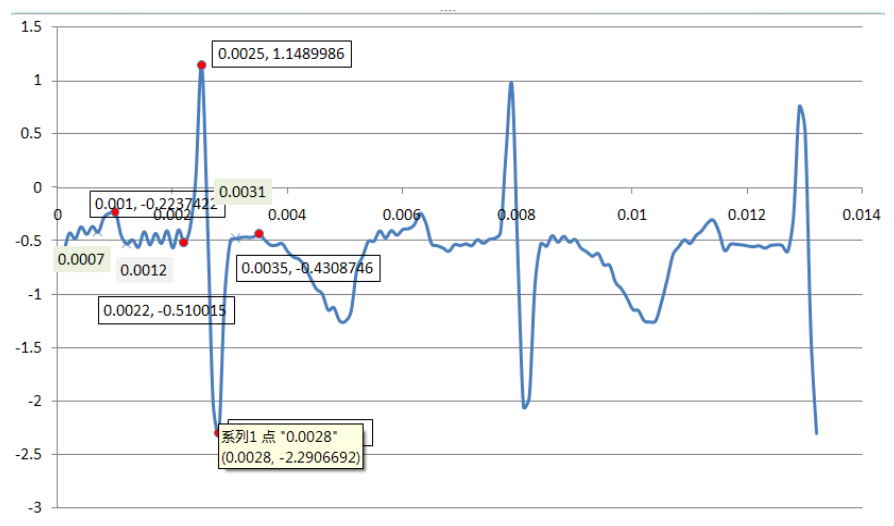

Figure 14. Sample of ECG signal.

Amplitude

$\mathrm{P}=-0.2237422$

$\mathrm{Q}=-0.510015$

$\mathrm{R}=-1.1489986$

$\mathrm{S}=-2.2906692$

$\mathrm{T}=-0.4308746$

Duration

$\mathrm{P}$ wave $=$ difference of $\mathrm{x}$-axis values

$0.0012-0.0007=0.0005$

QRS complex (considered as one complete waveform)

$\mathrm{Q}(\mathrm{x}$-axis $)=0.0022$

$\mathrm{S}(\mathrm{x}$-axis $)=0.0028$

QRS duration: $0.0028-0.0022=0.0006$

$\mathrm{T}$ wave $=$ difference of $\mathrm{x}$-axis values

$0.0037-0.0031=0.0006$

Gradient

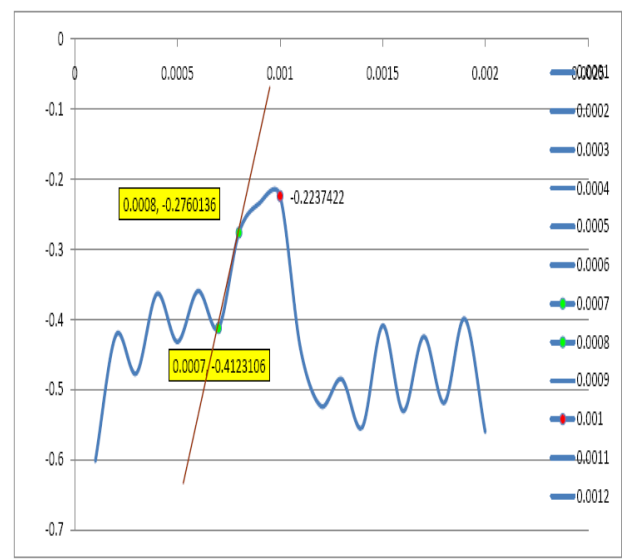

Figure 15. Pre-grad P.

$$
\begin{gathered}
\mathrm{m}=\frac{y_{2}-y_{1}}{x_{2}-x_{1}} \\
\text { Pre }-\operatorname{gradP}=\frac{-0.276 .136+.04123106}{0.0008-0.0007} \\
=\frac{0.13629}{0.0001}
\end{gathered}
$$

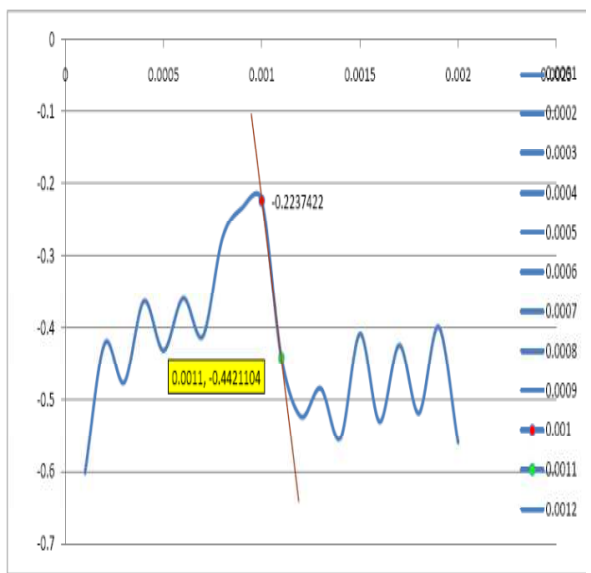

Figure 16. Post-grad P.

$$
\begin{aligned}
\mathrm{m}=\frac{-0.2237422+0.4421104}{0.001-0.0011} \\
\begin{aligned}
\text { Post }- \text { gradP } & =\frac{0.2183682}{-0.0001} \\
& =-2184
\end{aligned}
\end{aligned}
$$

This computation to find pre-gradient and post-gradient of other waves applied the same method as above.

Polarity

Definition: If the waveform is positive, then correspond- 
ing peak is set as 1 . If the waveform is negative, then the peak is set as 0 . Positive is referred to same pattern as the normal waveform, while the shape oppose the corresponding normal waveform is denoted as negative. In this case, polarity for each waveform assigned as below

$$
\begin{gathered}
\mathrm{P}=1 \\
\mathrm{QRS}=1 \\
\mathrm{~T}=1
\end{gathered}
$$

Data then been arranged using Microsof Excel and after normalization, the data then be fed as an inputs to neural network pattern recognition system. This normalization is important to ensure the values of the data are in between the certain range. Thus, it is easier to train the neural networks efficiently as well as easier for the network system to learn. The network will learn input characteristics and attributes of the corresponding waveforms. If for example the characteristics of $\mathrm{P}$ wave are given to the network for training, the target of $\mathrm{P}$ wave will be put as 1 and the other is 0 . Once the $\mathrm{P}$ wave is correctly detected, thus the real peak of $\mathrm{P}$ is easily obtained by the highest peak of that waveform. This is same goes to other waveforms.

\section{Result}

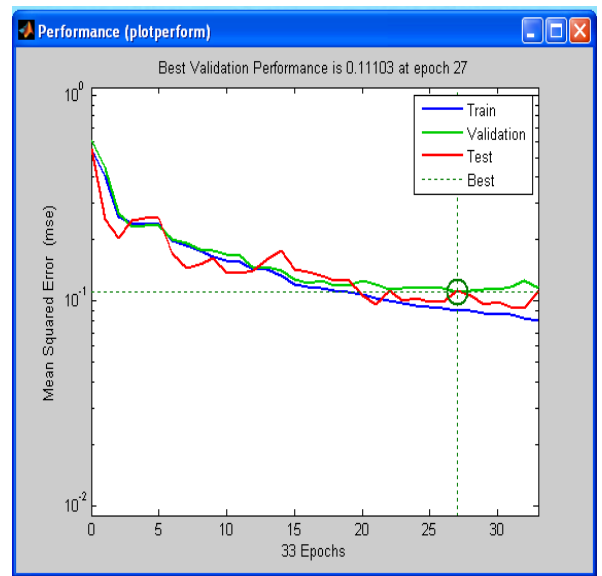

Figure 17. Network performance (MSE vs number of epochs).

Network's configuration has been detail identified with 80 hidden neurons, 2 layers feed forward, trained with scaled conjugate gradient back propagation (trainscg) with sigmoid type of hidden nodes and output neurons. 49 samples have been used with 5 input features, described as amplitude, duration, pre-gradient, post-gradient and polarity. The performance of the network is evaluated in mean squared error and confusion matrices. Network will be retrained to achieve as per desired target.

Simulation result showing that training and testing performance keep decreasing. In other words, it proved that the network is learning. During testing, it tried to achieved the target approximately as what has been trained. A part from that, it is seen that best validation performance, which is the smallest difference of desired target with the network output is at $1.1103 \times 10^{-1}$ after going through 27 iterations. At this point, the network possessed the ability to generalize very well after performance becomes minimized to the goal.

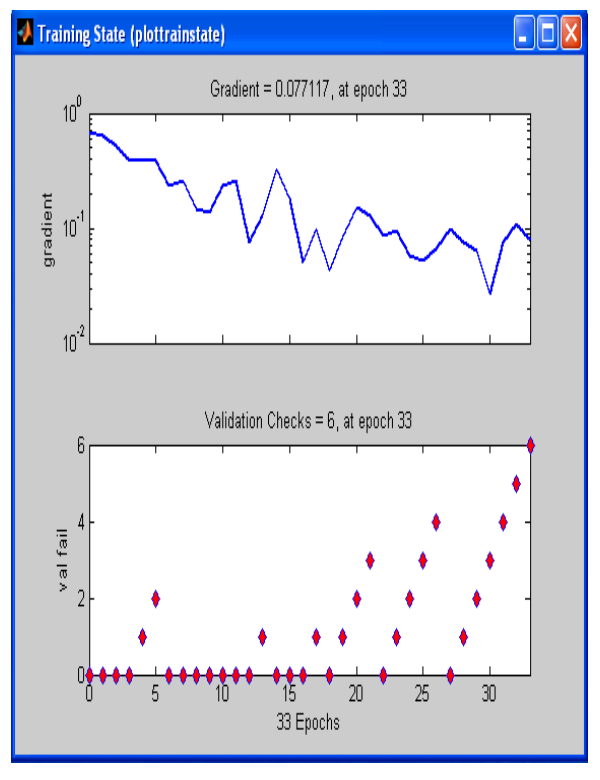

Figure 18. Network training state.

Result above presented training state of the network. The plot depicted the training state of the network from a training record. The minimum gradient reached at epochs 33 at a value of $7.77117 \times 10^{-2}$. Validation checks are at 6 also occurred during 33th iteration. The network has been well trained and learning to classify respective inputs to the corresponding target. It stops when minimum gradient has been reached to avoid network from overfitting and becomes uncontrolled. In neural network computing, validation is used to ensure the network able to generalize respective inputs mapping to the corresponding target. Validation will halt the training when generalization stops improving.

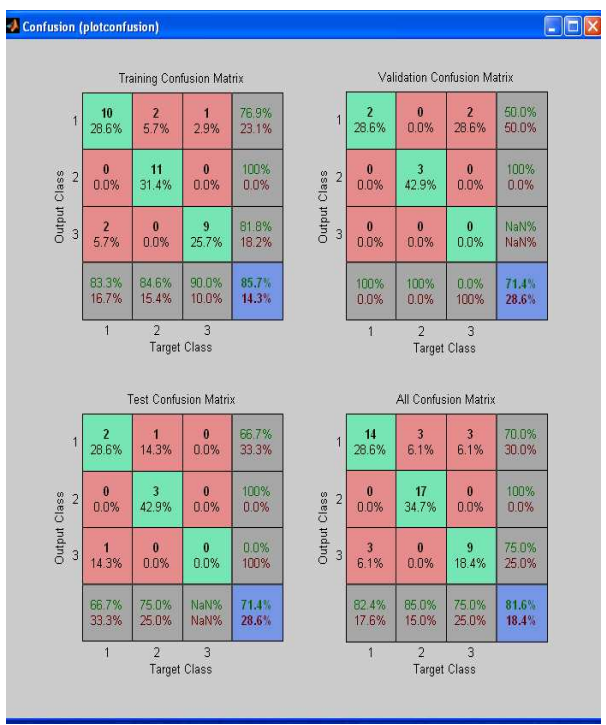

Figure 19. Confusion plot matrix. 
A confusion matrix depicted clearly the actual versus predicted class values. It is also presented the classes which are correctly classified and misclassified. Thus, it is enabling us to see how well the model predicts the outcomes [9]. There are three waveforms that need to be recognized by neural networks, which is waveform of $\mathrm{P}, \mathrm{QRS}$ and $\mathrm{T}$. The real peak is detected by the highest peak of the waveform. This is different for QRS. Once the QRS waveform is correctly identified by neural network system, thus it is known that the highest peak should be the real peak of $R$, the peak before is $\mathrm{Q}$ and the peak after the $\mathrm{R}$ peak should be $\mathrm{S}$ peak. 35 dataset has been used for training purposes, 7 for validation and 7 for testing phase. Based on the result above, it showed that during training session, there are 30 set of data are correctly classified and only 5 data are misclassified. In validation, 5 data are correctly classified out of 7 while during testing phase, 5 data too are correctly classified accordingly while twice of misclassification happened. Several factors contribute to network's improvement will be included in a discussion part. The percentage accuracy of each process (training, validation and testing) is also can be seen in the confusion plot matrix above. Overall network performance showing that 40 data are correctly classified as desired while 9 data are misclassified. It performed good classification with total accuracy of $81.6 \%$.

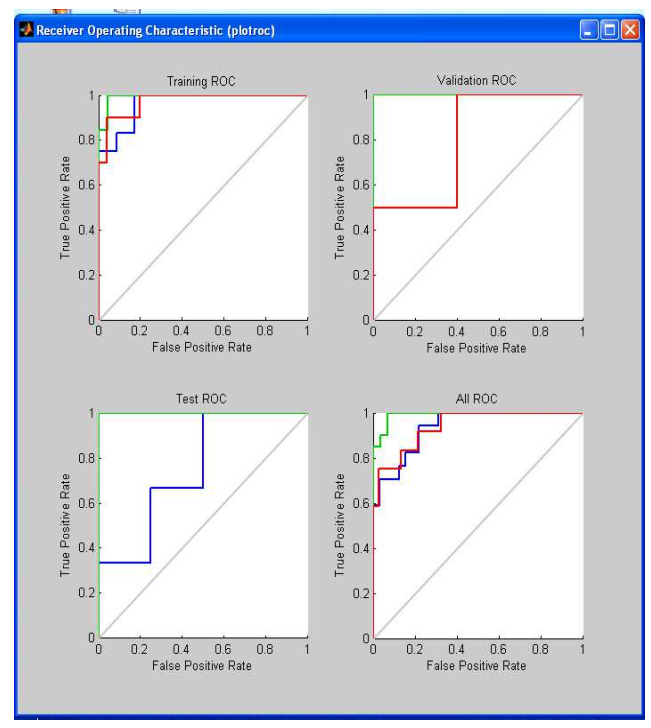

Figure 20. Receiver Operating Characteristic (ROC).

Above is shown the outcome of receiver operating characteristic (ROC) for this project. The ROC curve acts as fundamental tools for diagnostic evaluation for positive test which plotted true positive rate vs false positive rate [9], [10]. It reflects to a sensitivity and specificity of the network. True positive rate referred to real peak signals correctly identified as its real peak while false positive rate referred to non real peak of the signals incorrectly identified as real peak.

\section{Discussion}

The result for this project cannot achieve $100 \%$ or very high percentage of accuracy since small dataset was used. Thus, some recommendations could be suggested to improve network's performance in order to obtain approximately accurate outputs. There are including increase the numbers of hidden neurons and retrain the network several times. Furthermore, use larger data set so that the network will learn more and expose to enough trainings. Do try a different training algorithm as well as adjust the initial weighs and biases to new values. Then, train the networks again for several times until it reaches the desired target. In addition, it is recommended to perform automated features extraction using respective software such as LabVIEW or etc for accuracy and quick extraction purposes.

\section{Conclusion}

Neural network pattern recognition is suitable software with high ability to classify certain input patterns into a corresponding output target with overall accuracy of $81.6 \%$. The training accuracy is $85.7 \%$, validation accuracy is $71.4 \%$ while the testing accuracy is $71.4 \%$ too. It can be concluded that the real peak of ECG signals can be identified by training the network accordingly.

\section{Acknowledgement}

The appreciation goes to absolutely my main supervisor, Dr. Syed Sahal Nazli Alhady for provides endless help including motivation and guidance and also not forget to co-supervisor as well as field supervisor for some supported ideas directly or indirectly. My deepest gratitude then extends to Ministry of Science, Technology and Innovation, government of Malaysia for willing to sponsor me throughout my study, without the scholarship, this project might face difficulty and could not be accomplished in a comfortable manner. Thank you very much to those involved.

\section{References}

[1] A website on http://biology.about.com/library/organs/heart/blatrionode.ht $\mathrm{m}$, image courtesy of Carolina Biological Supply / Access Excellence.

[2] Chung, M.K, and Rich, M.W. Introduction to the cardiovascular system. Alcohol Health and Research World14 (4):269-276, 1990.

[3] Information, http://biology.about.com/od/humananatomybiology/ss/heart anatomy2.htm

[4] Mazhar B.Tayel, Mohamed E.El-Bouridy, ECG images classification using features extraction based on wavelet transformation and neural network, AIML 06 International Conference, 13-15 June 2006, Sharm El Sheikh, Egypt, 105-107.

[5] Rajesh Ghongade, Vishwakarma, Dr. Babasaheb, A brief 
Performance Evaluation of ECG Feature Extraction Techniques for Artificial Neural Network Based Classification.

[6] Christos Stergiou and Dimitrios Siganos, A Report on Neural Networks.

[7] Simon Haykin, A book of Neural Networks A Comprehensive Foundation, Second Edition, 1999.

[8] International journals on WSEAS Transactions on Systems, Issue 1, Volume 4, January 2005, ISSN 1109-2777, paper titled $\mathrm{P}, \mathrm{Q}, \mathrm{R}, \mathrm{S}$, and $\mathrm{T}$ peaks recognition of $\mathrm{ECG}$ using
MRBF with selected features, 137.

[9] M.P.S Chawla, Department of Electrical Engineering, Indian Institute of Technology, Roorkee, 247667 India, Parameterization and R-peak error estimations of ECG Signals using Independent Component Analysis, Computational and Mathematical Methods in Medicine, Vol. 8, No. 4, December 2007, 263-285.

[10] Exercise on Artificial Neural Networks, Information on rad.ihu.edu.gr/fileadmin/labsfiles/decision_support_systems /lessons/neural_nets/NNs.pdf. 